\title{
COVID-19 e Doença Cardiovascular: Consequências Diretas e Linhas de Investigação
}

\author{
COVID-19 and Cardiovascular Disease: Direct Consequences and \\ Lines of Investigation
}

Yasmin Mamade (https://orci.org/0000-0002-8811-6272), Inês Mendes (https://orci.org/0000-0002-4314-4376), Sílvia Balhana (https://orci.org/0000-0003-0818-1355), Carla Sofia Pereira (https://orci.org/0000-0003-1592-0806), Mafalda Vasconcelos (https://orci.org/0000-0001-8818-3697), Ana Patrícia Moreira (https://orci.org/0000-0002-1072-7364), Francisco Araújo (https://orci.org/0000-0002-6224-4866)

\section{Resumo:}

A infeção por SARS-CoV-2 não deixou ninguém incólume ou indiferente. Trouxe implicações profundas na vida diária e na saúde individual, mas também a nível económico e social. A nível da doença cardiovascular, a repercussão da COVID-19 foi enorme. Muito há ainda por saber e a informação é muitas vezes contraditória. Este artigo pretende apontar linhas de investigação que poderão ser úteis para encontrar respostas na relação entre a infeção a SARSCoV-2 e a doença cardiovascular. Exploramos aquilo que se sabe sobre os efeitos diretos da doença, abordando os mecanismos fisiopatológicos, quer sejam a agressão celular direta, a tempestade inflamatória, a angiopatia trombótica, a hipóxia ou a disfunção de lipoproteínas.

Palavras-chave: COVID-19; Doenças Cardiovasculares; Infecções por Coronavírus.

\section{Abstract:}

SARS-COV-2 infection has left no one unscathed or indifferent. It has had profound implications for personal daily lives and health, but also at a broader economic and social level. In terms of cardiovascular disease, COVID-19 had a huge impact. Much remains unknown and the information is often contradictory. This article aims to point out lines of investigation that may be useful to find answers about the link between SARS-COV-2 infection and cardiovascular disease. We explore the known direct effects of the disease, addressing the pathophysiological mechanisms, whether it is the direct cell aggression, inflammatory storm, thrombotic angiopathy, hypoxia or lipoprotein dysfunction.

Keywords: Cardiovascular Diseases; Coronavirus Infections; COVID-19.

Serviço de Medicina Interna, Hospital Beatriz Ângelo, Loures, Portugal

https://revista.spmi.pt - DOI:10.24950/R/110/20/3/2020

\section{Introdução}

A pandemia COVID-19 apanhou-nos a todos de surpresa, alterou as nossas vidas e afetou milhões de pessoas no mundo inteiro. São considerados doentes de risco para complicações relacionadas com a infeção por COVID-19, os doentes com doença cardiovascular estabelecida ou com fatores de risco vascular como a diabetes ou a hipertensão arterial. Que relação tem a COVID-19 com o sistema cardiovascular e que implicações poderá ter na saúde dos nossos doentes? (Fig. 1) Propomo-nos avaliar as repercussões cardiovasculares da infeção por SARS-CoV-2, explorando potenciais linhas de investigação sobre os mecanismos fisiopatológicos que justificam a multimorbilidade cardiovascular.

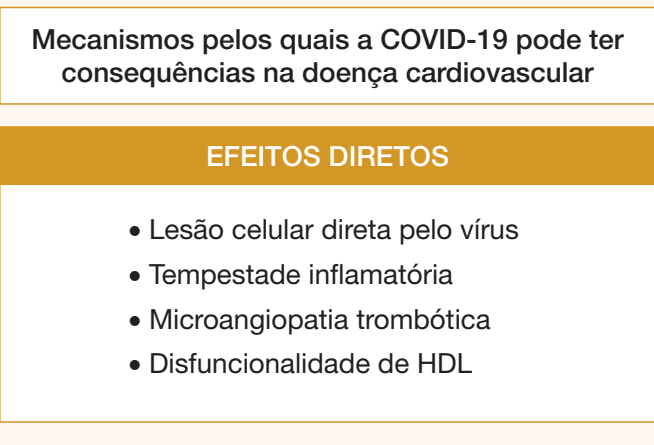

Figura 1: Potenciais implicações da COVID-19 na doença cardiovascular.

\section{ASPECTOS EPIDEMIOLÓGICOS, COMORBILIDADES E} RISCO VASCULAR

Shi et al demonstraram, analisando retrospetivamente 416 doentes internados por COVID-19 em Wuhan, no epicentro da pandemia, que existe uma associação entre lesão miocárdica (definida pela elevação da troponina I de alta sensibilidade) e o prognóstico da doença. Nos cerca de $20 \%$ de pacientes em que ocorreu lesão do miocárdio, houve um 
incremento da necessidade de ventilação mecânica invasiva (58\% vs 14,7\% nos doentes sem lesão miocárdica) e da mortalidade (51,2\% vs 4,5\%). Os doentes com lesão miocárdica eram mais velhos e tinham mais comorbilidades, nomeadamente maior prevalência de hipertensão arterial (HTA). ${ }^{1}$

Noutro estudo realizado na China, 1590 doentes admitidos por COVID-19 foram avaliados tendo como objetivo primário a admissão em cuidados intensivos, a necessidade de ventilação mecânica ou a morte. As comorbilidades tiveram impacto na ocorrência de objetivo primário, sendo a HTA equivalente à diabetes com uma taxa de risco relativo $(\mathrm{RR})$ de 1,6, inferior à doença pulmonar obstrutiva crónica (RR 2.3) ou cancro (RR 3.5). A idade média dos doentes era 49 anos e a maioria (58\%) eram mulheres. ${ }^{2}$ Destacamos também que $16 \%$ dos doentes tinham HTA, sendo que a prevalência desta doença na China é de $23 \%$. $^{3}$

Comorbilidades como a HTA e a diabetes não parecem ser um fator de risco para a infeção por coronavírus, mas conferem maior risco de complicações da doença. Na Europa, numa das maiores séries publicadas até a data, são descritos 1591 doentes admitidos em unidade de cuidados intensivos na Lombardia, com idade média 63 anos. Maioritariamente, eram homens (82\%), estando a HTA presente em $49 \%$ dos doentes e a doença cardiovascular em $21 \% .^{4}$

\section{APRESENTAÇÃO DA DOENÇA CARDIOVASCULAR NA COVID-19}

A apresentação clínica da infeção por SARS-CoV-2 é maioritariamente dominada por sintomas respiratórios, mas podem desenvolver-se complicações cardiovasculares, eventualmente como causa de morte. Até ao momento foram reportadas como possíveis manifestações, a miocardite, a síndrome coronária aguda, a insuficiência cardíaca descompensada, o tromboembolismo pulmonar, o choque cardiogénico e até mesmo infeção num coração transplantado. ${ }^{5} \mathrm{Na}$ COVID-19 ocorre um aumento das necessidades metabólicas, com diminuição progressiva na contração e reserva cardíacas, alterações responsáveis pelas apresentações cardiovasculares agudas ou descompensação de patologias crónicas. Uma das características da infeção por SARS-CoV-2 é a inflamação sistémica grave, capaz de produzir efeitos prócoagulantes ou uma disrupção auto-imune, podendo ambos os mecanismos condicionar a lesão miocárdica aguda. ${ }^{6} \mathrm{~A}$ miocardite aguda é uma complicação claramente reconhecida das infeções virais. Na COVID-19 a miocardite tem sido referida como o modo de apresentação da doença, apesar de (na maioria das vezes) não se ter comprovado o envolvimento miocárdico pelo vírus. Até à data, só casos clínicos isolados forneceram informação sobre a anatomopatologia do tecido miocárdico de doentes com COVID-19, o que nos impede de retirar conclusões definitivas. ${ }^{7}$ A prevalência real desta complicação ainda é desconhecida. ${ }^{8}$ Não estando completamente esclarecido o mecanismo por detrás da lesão, sabemos que se caracteriza por dor torácica, alterações eletrocardiográficas (como a elevação difusa do segmento ST) e elevações da troponina de alta sensibilidade, podendo ou não ser acompanhadas de febre e sintomas respiratórios. Numa coorte de 150 doentes infectados, com 68 mortes, 7\% destas foram atribuídas a miocardite com choque cardiogénico, e noutros $33 \%$ dos casos a miocardite pode ter representado um papel importante na morte do doente. ${ }^{9}$

As infeções virais estão associadas a disfunção metabólica, inflamação miocárdica e activação do sistema nervoso simpático. Todos eles são factores predisponentes a arritmias. Num estudo com 138 doentes hospitalizados com COVID-19, 16,7\% desenvolveram arritmias (fibrilhação auricular, bloqueios de condução, taquicardia ou fibrilhação ventricular). ${ }^{10} \mathrm{~A}$ incidência de arritmias é superior nos doentes mais graves, como os que necessitam de cuidados intensivos. ${ }^{11} \mathrm{~A}$ fibrilhação ventricular pode ser a apresentação inicial da infeção por SARS-CoV-2. Guo et al, demonstraram que em doentes com COVID-19 estratificados pelos níveis séricos de troponina, as arritmias ventriculares malignas eram duas vezes mais frequentes (11,5\% vs 5,2\%) na presença de níveis elevados de troponina.12 Assim, pensa-se que as arritmias possam ser causadas por lesão direta do cardiomiócito ou das células do sistema de condução, agravamento de uma doença miocárdica de base, alterações hidroeletrolíticas, stress adrenérgico ou isquémia por síndrome coronária aguda. ${ }^{9}$ Fármacos utilizados com frequência na terapêutica da COVID- 19 estão associados a maior risco de arritmia. Abordaremos este assunto posteriormente.

Está também demonstrado o risco aumentado de enfarte agudo do miocárdio após infeções respiratórias agudas, por vírus influenza ou outras espécies de coronavírus. ${ }^{13}$ A tempestade inflamatória desencadeada pela COVID-19 pode aumentar o risco de rutura de placas coronárias favorecendo a ocorrência de síndrome coronária aguda. ${ }^{12}$ Num estudo italiano com 28 doentes infetados por SARS-CoV-2 e enfarte agudo de miocárdio com supra do segmento ST, a angiografia coronária não revelou uma lesão culpável em 39,3\% dos casos. ${ }^{14}$ Para justificar esta mímica de apresentação como enfarte do miocárdio com artérias coronárias não obstruídas (MINOCA), são colocadas as hipóteses de enfarte tipo 2, miocardite, cardiomiopatia por stress, disfunção endotelial e estado de hipercoagulabilidade com trombose microvascular. ${ }^{9,15}$ A insuficiência cardíaca foi descrita em cerca de um quarto dos indivíduos com COVID-19, podendo ser atribuída à exacerbação da doença cardiovascular de base ou a uma nova manifestação de cardiomiopatia. Aproximadamente metade das vítimas mortais tinham sinais de insuficiência cardíaca, em comparação com 12\% dos sobreviventes. ${ }^{16} \mathrm{Em}$ doentes mais idosos e com doença cardiovascular conhecida, é frequente a hipertrofia ventricular e disfunção diastólica, sendo estes doentes mais propensos a desenvolver edema agudo do pulmão. ${ }^{9}$ 
A insuficiência cardíaca direita pode ser observada em contexto de hipertensão pulmonar em doentes com acute respiratory distress syndrome (ARDS) e/ou tromboembolismo pulmonar. Uma análise de 120 doentes com COVID19 demonstrou uma elevação dos níveis de NT-proBNP em $27,5 \%$ dos casos, indicando que os efeitos da lesão cardiovascular na estabilidade sistémica são importantes e não devem ser ignorados. ${ }^{14}$

Os doentes infetados com SARS-CoV-2 estão predispostos a desenvolver um estado de hipercoagulabilidade graças à imobilização prolongada, lesão endotelial e inflamação vascular. Em ambiente de cuidados intensivos quase um terço dos doentes registaram a ocorrência de eventos trombóticos venosos ou arteriais, sendo o tromboembolismo pulmonar o evento mais frequente. ${ }^{17}$

A incidência de tromboembolismo pulmonar nestes doentes é superior ao observado antes da pandemia em doentes com condições de gravidade semelhante ou infeções por influenza. ${ }^{9}$ O estado pró-trombótico e a disfunção endotelial provocadas pela infeção também parecem aumentar a probabilidade de acidente vascular cerebral (AVC). Beyrouti et al descreveram uma série de seis casos de doentes com COVID-19 com AVC isquémico: três doentes com enfartes multiterritorial e com trombose venosa profunda concomitante; e dois doentes com AVC isquémico apesar de terapêutica anticoagulante profilática. ${ }^{18}$ Oxley et al também reportaram uma série de casos (5 doentes de 33 a 49 anos) que sofreram AVC isquémico por oclusão de grandes vasos. ${ }^{19}$

\section{MECANISMOS FISIOPATOLÓGICOS DE LESÃO CAR- DIOVASCULAR NA COVID - LINHAS DE INVESTIGAÇÃO}

As razões para o maior risco de morte e complicações cardiovasculares nos doentes COVID-19 permanecem em grande parte por esclarecer, mas admitimos que em muitos, a causa será provavelmente multifatorial. Destacamos alguns mecanismos e potenciais linhas de investigação para explicar a fisiopatologia da doença cardiovascular na infeção por SARS-CoV-2.

\section{Lesão direta do coronavírus a nível celular}

A enzima conversora da angiotensina 2 (ECA2), envolvida na regulação da pressão arterial através da inibição fisiológica da ativação do sistema renina-angiotensina-aldosterona (SRAA), é o principal recetor do SARS-CoV-2. A ECA2 é amplamente expressa no endotélio vascular, epitélio respiratório, monócitos alveolares e macrófagos assim como em vários outros órgãos. A interação entre o vírus e a ECA2 foi proposta como fator potencial de infecciosidade. O vírus replica-se ativamente nas vias aéreas, gerando uma carga viral secundária que irá provocar lesão nos tecidos que expressam a ECA2, nomeadamente coração, rim, trato gastrointestinal e circulação periférica distal.20,21 Em autópsias realizadas a doentes falecidos com complicações sistémicas da COVID-19, demonstrou-se a supra-expressão do recetor ACE2 a nível renal, com inclusão de partículas COVID-19 no epitélio tubular e podócitos, e diferentes graus de lesão sobretudo a nível do tubo contornado proximal. ${ }^{22}$ Outros dados anatomopatológicos demonstraram a inclusão de partículas de COVID-19 em células endoteliais, associadas a inflamação difusa, uma endotelite portanto... A disfunção endotelial associada, pode contribuir para o agravamento de isquémia associada à vasoconstrição, inflamação dos tecidos periféricos e o desenvolvimento de um estado pró-coagulante. Especula-se se fármacos protetores do endotélio como as estatinas poderiam ter um papel na proteção das complicações por COVID-19. ${ }^{23,24}$ Nos Estados Unidos, autópsias realizadas a 23 doentes cuja causa de morte foi atribuída à COVID-19, mostraram com frequência, lesão multifocal dos cardiomiócitos sem infiltrado inflamatório, pericardite linfocítica em três casos e miocardite linfocítica num caso isolado. ${ }^{25}$

\section{Tempestade inflamatória}

A gravidade da COVID-19 está relacionada não só com a toxicidade viral direta, mas também com a desregulação e hiperatividade imune, que se traduzem na produção descontrolada de citocinas e quimiocinas inflamatórias, fenómeno designado por tempestade inflamatória ou tempestade de citocinas. ${ }^{15,26}$ A COVID-19 apresenta também semelhanças com a síndrome hemofagocítica secundária, nomeadamente no perfil de citocinas, marcadores serológicos e sintomas. Esta síndrome é potencialmente desencadeada por infeções virais e, embora o mecanismo fisiopatológico não esteja completamente esclarecido, pensa-se que os níveis aumentados de moléculas inflamatórias contribuam para a destruição tecidular por hiperatividade da linhagem macrocítica-macrofágica. Este fenómeno pode ser um dos grandes responsáveis pela lesão tecidular que conduz à síndrome de dificuldade respiratória aguda (ARDS) e à lesão de outros órgãos, nomeadamente do sistema cardiovascular. ${ }^{27}$ Uma das citocinas implicadas no desenvolvimento da síndrome hemofagocítica é a IL-6, cujos níveis séricos mais elevados relacionam-se com risco de ARDS e de mortalidade. ${ }^{28}$ Outros marcadores inflamatórios, como a ferritina e outras citocinas e quimiocinas inflamatórias (GCSF, IP-10, MCP-1, TNF- $\alpha$ ) estão relacionadas com a gravidade da doença, sugerindo que a inflamação sistémica pode constituir um fator significativo no desenvolvimento de lesão multiorgânica. ${ }^{29,30}$ É ainda importante referir que na COVID-19 ocorre um aumento da contagem de neutrófilos e leucócitos, e uma redução marcada do número de linfócitos T CD4+, T CD8+, T reguladores, T de memória, NK e B. ${ }^{27}$ Este fenómeno de linfopenia ocorre provavelmente por sequestração destas células e traduz um compromisso do sistema imunitário. ${ }^{26}$ Exames anatomopatológicos mostraram atrofia esplénica, com necrose e hemorragia, bem como uma redução muito significativa do número de linfócitos e neutrófilos no baço. Há também uma depleção 
de linfócitos nos gânglios linfáticos. ${ }^{15}$ No contexto de inflamação sistémica, as células T ativadas e os macrófagos podem infiltrar o miocárdio, em alguns casos provocando lesão cardíaca grave. A lesão miocárdica aguda, inicia-se geralmente duas semanas após o início de sintomas, pelo que se pensa que a imunidade adaptativa mediada por células T ou a imunidade inata desregulada tenham um papel no seu desenvolvimento. É possível que a tempestade de citocinas despolete uma miocardite autoimune previamente subclínica, ou que o dano miocárdico e/ou o mimetismo molecular (por exemplo, por semelhança entre os antigénios virais e a cadeia pesada de miosina) iniciem uma reação auto-imune de novo. ${ }^{15}$ Verificou-se uma correlação positiva entre o aumento de marcadores inflamatórios e a lesão miocárdica, o que é consistente com a hipótese de a hiperinflamação ser causa de disfunção cardíaca. ${ }^{31}$ Alguns estudos experimentais sugerem que a IL-6 e as citocinas relacionadas com a síndrome de libertação de citocinas têm propriedades aterogénicas e se relacionam com o desenvolvimento de fibrose cardíaca e insuficiência cardíaca. In vitro, demonstrou-se que as citocinas aumentam as moléculas de adesão expressas em células humanas endoteliais e que as moléculas de colesterol LDL oxidadas induzem a libertação de IL-6 pelos macrófagos, a formação de placas ateroscleróticas e a progressão de aneurismas da aorta abdominal. A sobre-regulação do recetor de IL-6, por sua vez, causa remodelagem vascular e hipertensão arterial pulmonar. ${ }^{15}$ Não está ainda claro se a elevação dos níveis de IL-6 e a tempestade de citocinas no contexto da COVID-19 são fenómenos transitórios ou sustentados no tempo. ${ }^{15}$ Por último, o sistema renina-angiotensina-aldosterona pode também ter um papel importante. Como referido, o SARS-CoV-2 infeta as células ligando-se à ECA2, a enzima responsável pela clivagem da angiotensina II em angiotensina 1-7, tendo esta última efeitos vasodilatadores e anti-inflamatórios. A infra-regulação da ECA2 pelo vírus pode aumentar a estimulação pela angiotensina II, contribuindo assim para a reação hiper-inflamatória da COVID-19. ${ }^{31}$

\section{Hipóxia grave}

Até $15 \%$ dos doentes internados por COVID-19 desenvolvem pneumonia, ARDS, lesão miocárdica, lesão renal ou disfunção multiorgânica entre os 7-10 dias após a admissão hospitalar. Uma percentagem considerável de doentes apresenta agravamento progressivo da hipoxemia, por vezes com uma evolução rápida. ${ }^{32} \mathrm{Na}$ província de Hubei, 60\%-70\% dos pacientes admitidos nas unidades de cuidados intensivos (UCl) tinham ARDS. ${ }^{10,33} \mathrm{~A}$ hipóxia induzida pelo aumento de cálcio intracelular, pode levar à apoptose dos miócitos, contribuindo para o aumento da morbilidade cardiovascular. ${ }^{34}$ Também está bem estabelecido o nexo de causalidade entre a insuficiência respiratória e o enfarte tipo 2, em particular quando se gera um desequilíbrio entre o aporte e as necessidades de oxigénio no tecido miocárdico, mesmo na ausência de relação directa com a doença aterosclerótica subjacente. ${ }^{35}$ Ao contrário da apresentação "típica" de ARDS (definição de Berlim), nos doentes com COVID-19 existe uma dissociação entre um pulmão mecanicamente funcional (com objetivação de boas compliances) e a gravidade da hipoxemia. ${ }^{36}$ Importa assim rever o papel que a microangiopatia trombótica poderá ter neste contexto.

\section{Microangiopatia vascular}

O risco de eventos tromboembólicos está bem estabelecido em doentes hospitalizados por doença infeciosa aguda, bem como nos doentes acamados, com outras comorbilidades (ex. neoplasia ativa, diabetes, insuficiência cardíaca), ou nos doentes críticos. Existem múltiplos scores de avaliação de risco que permitem suportar a decisão quanto ao início de anticoagulação profilática nestes doentes.

A COVID-19 apresenta um risco tromboembólico aumentado, registando-se um número considerável de eventos de tromboembolismo venoso (TEV) e uma maior incidência de coagulação vascular disseminada (CID), o que confere a esta doença pior prognóstico. Comparando (em autópsias) as lesões pulmonares da COVID-19, com as que decorrem de doentes com influenza A ( $\mathrm{H} 1 \mathrm{~N} 1)$, constatou-se uma clara superioridade (9 vezes mais) na prevalência das alterações trombóticas a nível da microvasculatura nos doentes com infeção a SARS-CoV-2. ${ }^{24}$ A doença tromboembólica pode passar despercebida. Em 7 de 12 doentes (58\%) mortos com COVID-19, sem suspeita prévia de tromboembolismo, havia evidência de trombose venosa profunda na autópsia. ${ }^{37} \mathrm{Al}-$ guns autores defendem que os mecanismos subjacentes a este risco estão relacionados com a disfunção endotelial consequente à lesão direta do vírus, a mecanismos de supressão medular e à ativação do sistema hemofagocítico, com consequente libertação de citocinas inflamatórias, aumento da produção de trombina, ativação plaquetária e diminuição dos mecanismos anti-fibrinolíticos. ${ }^{38-40} \mathrm{~A}$ nível laboratorial as alterações mais comuns são: trombocitopenia ligeira, aumento dos d-dímeros e do fibrinogénio. Existem estudos que apontam para uma maior gravidade da doença em doentes onde se verifica prolongamento do tempo de protrombina (TP) e do INR, bem como nos que apresentam um tempo de tromboplastina parcialmente ativada (aPTT) reduzido. ${ }^{33,41}$ Num estudo realizado em 184 doentes admitidos em $\mathrm{UCl}$ por pneumonia a COVID-19, todos sob profilaxia para tromboembolismo, $31 \%$ tiveram evidência de evento tromboembólico venoso ou arterial agudo. Estes eventos foram definidos como embolia pulmonar, acidente vascular cerebral, enfarte do miocárdio ou embolia arterial periférica, sendo a embolia pulmonar o evento diagnosticado mais frequentemente (81\%). A par da idade, um TP superior a 3 segundos ou um aPTT superior a 5 segundos foram os melhores preditores de complicações. ${ }^{37}$ Num hospital em Wuhan, 8,7\% dos doentes internados com COVID-19 desenvolveram CID, verificando-se ainda que $71 \%$ 


\section{Doente com sintomas de COVID-19}

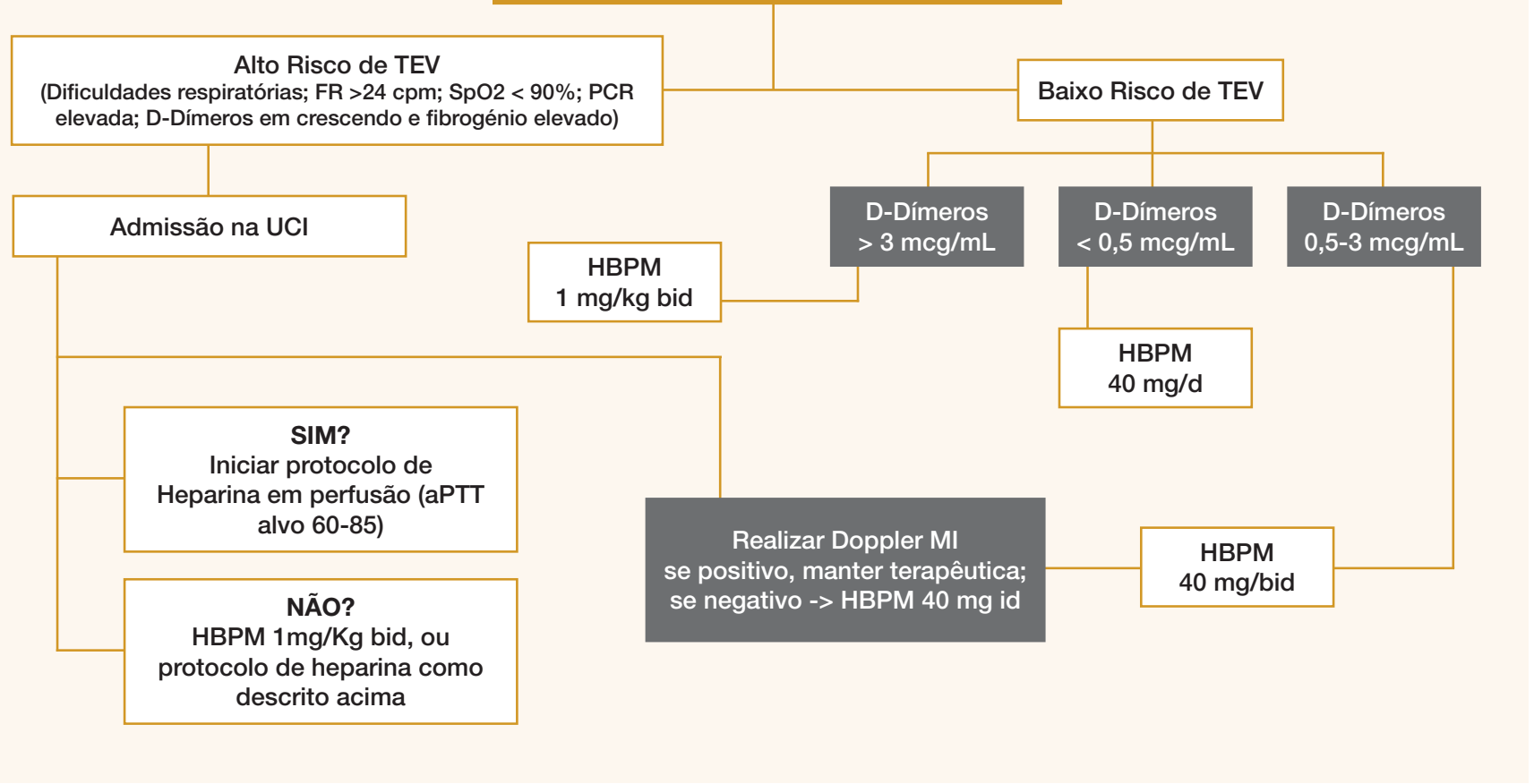

Figura 2: Protocolo hipocoagulação em doentes COVID-19.43

dos pacientes que faleceram preenchiam critérios para CID, comparativamente a $0,6 \%$ dos sobreviventes. ${ }^{39}$ Neste contexto, a utilização de anticoagulação profilática empírica está recomendada para a generalidade dos doentes. Este tema tem sido debatido intensamente; nas formas de apresentação graves, essa dose "profilática" pode ser duplicada. Em prevenção secundária, a dose recomendada de forma mais consensual é a mesma que a utilizada para os doentes não-COVID. Os cuidados habituais com o risco hemorrágico, peso e compromisso da função renal mantêm-se. ${ }^{42}$ Alguns centros adotaram como método de avaliação para a disfunção multiorgânica o score SIC (sepsis induced coagulopathy) que engloba o tempo de protrombina, contagem de plaquetas e o score de SOFA (sequential organ failure assessment). Também é utilizado como cut-off para o início de anticoagulação, nível de d-dímeros 6 vezes superior ao limite superior do normal. Num estudo chinês que envolveu 449 pacientes, a utilização de anticoagulantes apenas mostrou benefício na mortalidade aos 28 dias, nos pacientes que tinham COVID19 grave e um score SIC quatro ou mais vezes aumentado ( $p$ $=0,029)$ ou d-dímeros acima de seis vezes o limite do normal $(p=0,017) .38$ Atallah et al defendem um algoritmo (Fig. 2) de início de anticoagulação baseado na estratificação dos pacientes consoante o risco de TEV, valor dos d-dímeros e nos resultados do doppler dos membros inferiores. ${ }^{43}$ Contudo os estudos na área da hipocoagulação ainda são escassos, com amostras pequenas e seguimento a curto prazo; muito há ainda por saber sobre a verdadeira incidência dos eventos tromboembólicos e a eficácia da anticoagulação nestes doentes.

\section{Disfunção do HDL- colesterol na pneumonia viral}

As partículas lipoproteicas de colesterol de alta densidade (HDL) parecem ter benefícios que ultrapassam a sua ação predominante no transporte reverso de colesterol, tendo sido bem demonstradas ações anti-inflamatórias, antioxidantes e vaso protetoras.

No contexto de infeção viral, as HDL podem tornar-se disfuncionais, contribuindo para a ocorrência de complicações pulmonares, tendo sido demonstrada esta relação nas pneumonias associadas ao vírus influenza. ${ }^{44}$ Em modelos animais infetados com influenza A, as HDL apresentam menor atividade anti-inflamatória e antioxidante (traduzida por redução da atividade da paraoxonase e do fator ativador plaquetário acetil-hidrolase), e por outro lado revelando elevação da ceruloplasmina, um potente oxidante. ${ }^{45}$ Fármacos que modulam a função, níveis e características das HDL, poderão ser uma linha terapêutica na redução das complicações da COVID.

\section{Conclusão}

Desde os primeiros casos de infeção a SARS-CoV-2 já foram dados pequenos passos na perceção das implicações desta pandemia na doença cardiovascular, contudo apenas é conhecida a ponta do iceberg. Desde a hipoxia grave à tempestade inflamatória, sabe-se que a COVID-19 provoca 
alterações fisiopatológicas com grande impacto no sistema cardiovascular, prevendo-se um aumento da morbimortalidade dos nossos doentes. Existem ainda potenciais efeitos indiretos que não devem ser desconsiderados, estes serão alvo de estudo em artigos futuros. Só o tempo mostrará o peso deste novo vírus na doença cardiovascular.

\section{Responsabilidades Éticas}

Conflitos de Interesse: Os autores declaram a inexistência de conflitos de interesse na realização do presente trabalho.

Fontes de Financiamento: Não existiram fontes externas de financiamento para a realização deste artigo.

Confidencialidade dos Dados: Os autores declaram ter seguido os protocolos da sua instituição acerca da publicação dos dados de doentes.

Proteção de Pessoas e Animais: Os autores declaram que os procedimentos seguidos estavam de acordo com os regulamentos estabelecidos pelos responsáveis da Comissão de Investigação Clínica e Ética e de acordo com a Declaração de Helsínquia da Associação Médica Mundial. Proveniência e Revisão por Pares: Não comissionado; revisão externa por pares.

\section{Ethical Disclosures}

Conflicts of interest: The authors have no conflicts of interest to declare.

Financing Support: This work has not received any contribution, grant or scholarship

Confidentiality of Data: The authors declare that they have followed the protocols of their work center on the publication of data from patients.

Protection of Human and Animal Subjects: The authors declare that the procedures followed were in accordance with the regulations of the relevant clinical research ethics committee and with those of the Code of Ethics of the World Medical Association (Declaration of Helsinki).

Provenance and Peer Review: Not commissioned; externally peer reviewed.

(c) Autor (es) (ou seu (s) empregador (es)) e Revista SPMI 2020. Reutilização permitida de acordo com CC BY-NC. Nenhuma reutilização comercial. (C) Author(s) (or their employer(s)) and SPMl Journal 2020. Re-use permitted under CC BY-NC. No commercial re-use.

\section{Correspondence / Correspondência:}

Francisco Araújo - farausjoster@gmail.com

Serviço de Medicina Interna, Hospital Beatriz Ângelo, Loures, Portugal

Received / Recebido: 15/06/2020

Accepted / Aceite: 26/08/2020

Publicado / Published: 28 de Setembro de 2020

\section{REFERÊNCIAS}

1. Shi S, Qin M, Shen B, Cai Y, Liu T, Yang F, et al. Association of Cardiac Injury With Mortality in Hospitalized Patients With COVID-19 in Wuhan, China. JAMA Cardiol. 2020 (in press). doi:10.1001/jamacardio.2020.0950

2. Guan WJ, Liang WH, Zhao Y, Liang HR, Chen ZS, for the China Medical Treatment Expert Group for Covid-19. Comorbidity and its impact on 1590 patients with Covid-19 in China: A Nationwide Analysis. Eur Respir J. 2020 (in press). doi: 10.1183/13993003.00547-2020

3. Wang Z, Chen Z, Zhang L, for the China Hypertension Survey Investigators. Status of Hypertension in China: Results From the China Hypertension Survey, 2012-2015. Circulation. 2018;137:2344-56. doi: 10.1161/CIRCULATIONAHA.117.032380.

4. Grasselli G, Zangrillo A, Zanella A, Antonelli M, Cabrini L, Castelli A, et al. Baseline Characteristics and Outcomes of 1591 Patients Infected With SARS-CoV-2 Admitted to ICUs of the Lombardy Region, Italy. JAMA. 2020;323:1574-81. doi:10.1001/jama.2020.5394

5. Barison A, Aimo A, Castiglione V, Arzilli C, Lupón J, Codina P, et al. Cardiovascular disease and COVID-19: les liaisons dangereuses. Eur J Prev Cardiol. 2020 (in press). doi: 10.1177/2047487320924501.

6. Dixon DL, Van Tassell BW, Vecchié A, Bonaventura A, Talasaz AH, Kakavand $\mathrm{H}$,et al. Cardiovascular Considerations in Treating Patients With Coronavirus Disease 2019 (COVID-19). J Cardiovasc Pharmacol. 2020;75:359-67. doi: 10.1097/FJC.0000000000000836.

7. Sala S, Peretto G, Gramegna M, Palmisano A, Villatore A, Vignale D, et al. Acute myocarditis presenting as a reverse Tako-Tsubo syndrome in a patient with SARS-CoV-2 respiratory infection. Eur Heart J. 2020 (in press). doi:10.1093/eurheartj/ehaa286.

8. Inciardi RM, Lupi L, Zaccone G, Italia L, Raffo M, Tomasoni D, et al. Cardiac Involvement in a Patient With Coronavirus Disease 2019 (COVID-19). JAMA Cardiol. 2020 (in press).doi:10.1001/jamacardio.2020.1096

9. Boukhris M, Hillani A, Moroni F, Annabi MS, Addad F, Ribeiro MH, et al Cardiovascular implications of the COVID-19 pandemic: a global perspective. Can J Cardiol. 2020 (in press). doi: 10.1016/j.cjca.2020.05.018

10. Wang D, Hu B, Hu C, Zhu F, Liu X, Zhang J, et al. Clinical Characteristics of 138 Hospitalized Patients With 2019 Novel Coronavirus-Infected Pneumonia in Wuhan, China. JAMA. 2020;323:1061-9. doi:10.1001/ jama.2020.1585

11. Liu K, Fang YY, Deng Y, Liu W, Wang MF, Ma JP, et al. Clinical characteristics of novel coronavirus cases in tertiary hospitals in Hubei Province. Chin Med J. 2020 (in press). doi: 0.1097/CM9.0000000000000744.

12. Guo T, Fan Y, Chen M, Wu X, Zhang L, He T, et al. Cardiovascular Implications of Fatal Outcomes of Patients With Coronavirus Disease 2019 (COVID-19). JAMA Cardiol. 2020 (in press). doi: 10.1001/jamacardio.2020.1017

13. Kwong JC, Schwartz KL, Campitelli MA, Chung H, Crowcroft NS, Karnauchow $\mathrm{T}$, et al. Acute Myocardial Infarction after Laboratory-Confirmed Influenza Infection. N Engl J Med. 2018;378:345-53. doi: 10.1056/NEJMoa1702090

14. Stefanini GG, Montorfano M, Trabattoni D, Andreini D, Ferrante G, Ancona $M$ et al. ST-Elevation Myocardial Infarction in Patients with COVID-19: Clinical and Angiographic Outcomes. Circulation. 2020 (in press). doi:10.1161/ CIRCULATIONAHA.120.047525

15. Guzik TJ, Mohiddin SA, Dimarco A, Patel V, Savvatis K, Marelli-Berg FM, et al. COVID-19 and the cardiovascular system: implications for risk assessment, diagnosis, and treatment options.Cardiovasc Res. 2020 (in press). doi:10.1093/cvr/cvaa106

16. Zhou P, Yang XL, Wang XG, Hu B, Zhang L, Zhang W, et al. A pneumonia outbreak associated with a new coronavirus of probable bat origin. Nature. 2020;579:270-3.

17. Klok FA, Kruip MJ, van der Meer NJ, Arbous MS, Gommers DA, Kant $\mathrm{KM}$, et al. Incidence of thrombotic complications in critically ill ICU patients with COVID-19.]. Thromb Res. 2020 (in press). doi:10.1016/j. thromres.2020.04.013

18. Beyrouti R, Adams ME, Benjamin L, Cohen H, Farmer SF, Goh YY, et al Characteristics of ischaemic stroke associated with COVID-19. J Neurol Neurosurg Psychiatry. 2020 (in press). doi:10.1136/jnnp-2020-323586

19. Oxley TJ, Mocco J, Majidi S, Kellner CP, Shoirah H, Singh IP, et al. Large-Vessel Stroke as a Presenting Feature of Covid-19 in the Young. N Engl J Med. 2020;382:e60. doi:10.1056/NEJMc2009787

20. Cao W, Li T. COVID-19: towards understanding of pathogenesis. Cell Res. 2020;30:367-369. doi:10.1038/s41422-020-0327-4

21. Hamming I, Timens W, Bulthuis ML, Lely AT, Navis G, van Goor H. Tissue distribution of ACE2 protein, the functional receptor for SARS coronavirus. A first step in understanding SARS pathogenesis. J Pathol. 2004;203:6317. doi:10.1002/path.1570

22. Su H, Yang M, Wan C, Yi LX, Tang F, Zhu HY,et al. Renal histopathological analysis of 26 postmortem findings of patients with COVID-19 in China. 
Kidney Int. 2020 (in press). doi:10.1016/j.kint.2020.04.003

23. Varga Z, Flammer AJ, Steiger P, Haberecker M, Andermatt R, Zinkernagel AS, et al. Endothelial cell infection and endotheliitis in COVID-19. Lancet. 2020;395:1417-8. doi:10.1016/S0140-6736(20)30937-5

24. Ackermann M, Verleden SE, Kuehnel M, Haverich A, Welte T, Laenger F et al. Pulmonary Vascular Endothelialitis, Thrombosis, and Angiogenesis in Covid-19. N Engl J Med. 2020 (in press). doi:10.1056/NEJMoa2015432

25. Buja LM, Wolf DA, Zhao B, Akkanti B, McDonald M, Lelenwa L, et al. The emerging spectrum of cardiopulmonary pathology of the coronavirus disease 2019 (COVID-19): Report of 3 autopsies from Houston, Texas, and review of autopsy findings from other United States cities. Cardiovasc Pathol. 2020;48:107233. doi:10.1016/j.carpath.2020.107233

26. Zhu H, Rhee JW, Cheng P, Waliany S, Chang A, Witteles RM, et al Cardiovascular Complications in Patients with COVID-19: Consequences of Viral Toxicities and Host Immune Response [published correction appears in Curr Cardiol Rep. 2020;22:36]. Curr Cardiol Rep. 2020;22:32. doi:10.1007/s11886-020-01292-3

27. Tufan A, Avanoglu Güler A, Matucci-Cerinic M. COVID-19, immune system response, hyperinflammation and repurposing antirheumatic drugs. Turk J Med Sci. 2020;50:620-32. doi:10.3906/sag-2004-168

28. Wang W, He J, Lie p, Huang I, Wu S.. The definition and risks of cytokine release syndrome-like in 11 COVID19-infected pneumonia critically ill patients: disease characteristics and retrospective analysis. Med Rxiv 2020. doi: 10.1101/2020.02.26.20026989

29. Huang C, Wang Y, Li X, Ren L, Zhao J, Hu Y, et al. Clinical features of patients infected with 2019 novel coronavirus in Wuhan, China [published correction appears in Lancet. 2020]. Lancet. 2020;395:497-506. doi:10.1016/S0140-6736(20)30183-5

30. Ruan Q, Yang K, Wang W, Jiang L, Song J. Clinical predictors of mortality due to COVID-19 based on an analysis of data of 150 patients from Wuhan, China [published correction appears in Intensive Care Med. 2020]. Intensive Care Med. 2020;46:846-8. doi:10.1007/s00134-020-05991-x

31. Tomasoni D, Italia L, Adamo M, Inciardi RM, Lombardi CM, Solomon SD, et al. COVID 19 and heart failure: from infection to inflammation and angiotensin II stimulation. Searching for evidence from a new disease [published online ahead of print, 2020]. Eur J Heart Fail. 2020 (in press). doi:10.1002/ ejhf.1871

32. Rello J, Storti E, Belliato M, Serrano R. Clinical phenotypes of SARS-CoV-2: implications for clinicians and researchers. Eur Respir J. 2020;55:2001028. doi:10.1183/13993003.01028-2020
33. Guan WJ, Ni ZY, Hu Y, Liang WH, Ou CQ, He JX, et al. Clinical Characteristics of Coronavirus Disease 2019 in China. N Engl J Med. 2020;382:170820. doi:10.1056/NEJMoa2002032

34. Zheng YY, Ma YT, Zhang JY, Xie X. COVID-19 and the cardiovascular system. Nat Rev Cardiol. 2020;17:259-60. doi:10.1038/s41569-020-0360-5

35. Thygesen K, Alpert JS, Jaffe AS, Chaitman BR, Bax JJ, Morrow DA, et al. Fourth Universal Definition of Myocardial Infarction (2018). J Am Coll Cardiol. 2018;72:2231-64. doi:10.1016/j.jacc.2018.08.1038

36. Gattinoni L, Coppola S, Cressoni M. COVID-19 does not lead to a "typical" acute respiratory distress syndrome. Am J Respir Crit Care Med. 2020 (in press).

37. Wichmann D, Sperhake JP, Lütgehetmann M, Steurer S, Edler C, Heinemann A, et al. Autopsy Findings and Venous Thromboembolism in Patients With COVID-19.]. Ann Intern Med. 2020 (in press). doi:10.7326/M20-2003

38. Tang N, Bai H, Chen X, Gong J, Li D, Sun Z. Anticoagulant treatment is associated with decreased mortality in severe coronavirus disease 2019 patients with coagulopathy. J Thromb Haemost. 2020;18:1094-99. doi:10.1111/jth.14817

39. Tang N, Li D, Wang X, Sun Z. Abnormal coagulation parameters are associated with poor prognosis in patients with novel coronavirus pneumonia. $J$ Thromb Haemost. 2020;18:844-7. doi:10.1111/jth.14768

40. Xie Y, Wang X, Yang P, Zhang S. COVID-19 complicated by acute pulmonary embolism. Radiol Cardiothorac Imaging 2020;2:e200067-e200067

41. Kreuziger L, Lee A, Garcia D, Cuker A, Cushman M, Connors J. COVID-19 and VTE-anticoagulation. American Society of Hematology - resource for COVID-19 and coagulopathy 2020. Chicago: ASH; 2020.

42. Barnes GD, Burnett A, Allen A, Blumenstein M, Clark NP, Cuker A, et al. Thromboembolism and anticoagulant therapy during the COVID-19 pandemic: interim clinical guidance from the anticoagulation. J Thromb Thrombolysis. 2020 (in press). doi:10.1007/s11239-020-02138-z

43. Atallah B, Mallah SI, AlMahmeed W. Anticoagulation in COVID-19.Eur Heart J Cardiovasc Pharmacother. 2020 (in press). doi:10.1093/ehjcvp/ pvaa036

44. Gordon EM, Figueroa DM, Barochia AV, Yao X, Levine SJ. High-density Lipoproteins and Apolipoprotein A-I: Potential New Players in the Prevention and Treatment of Lung Disease. Front Pharmacol. 2016;7:323.

45. Van Lenten BJ, Wagner AC, Nayak DP, Hama S, Navab M, Fogelman AM. High-density lipoprotein loses its anti-inflammatory properties during acute influenza A infection. Circulation. 2001;103:2283-88. doi:10.1161/01. cir.103.18.2283 